\title{
EXAMINATION OF DENTIN SURFACE USING AFM (OUR EXPERIENCE)
}

\author{
Zdeňka Zapletalová1, Roman Kubínek ${ }^{2}$, Milan Vujjtek ${ }^{2}$, Radko Novotný3
}

Palacky University Olomouc, Faculty of Medicine, Czech Republic: 1st Clinic of Dentistry ${ }^{1}$, Department of Microscopy methods ${ }^{3}$; Palacky University Olomouc, Faculty of Science, Czech Republic: Department of Experimental Physics ${ }^{2}$

Summary: Atomic force microscopy (AFM) as one the technique of Scanning Probe Microscopy is useful for imaging of surface structure. This method can yield three-dimensional high-resolution topographic images of sample surfaces by using a scanning technique for conductors and insulators on atomic scale. It is based upon mapping of atomic-forces on a surface of an investigated sample. The method is useful not only in physics and chemistry; it can be also applied in biological fields. Special construction of AFM scanner enables to follow biological samples in liquid environments. Artifacts caused by dehydration of samples are removed this way. Dentin of human teeth is a vital hydrated tissue. It is strongly sensitive to dehydration and drying that are commonly used in preparation of samples in examinations by Scanning Electron Microscopy (SEM). We describe our experience in examination of dentin surfaces of extracted human third molars using contact method of AFM under moist conditions.

Key words: Atomic Force Microscopy (AFM); Dentin; Smear layer

\section{Introduction}

Scanning Probe Microscopy (SPM) is a set of experimental methods used in imaging of surface structures with subatomic resolution (8). Beside physics and chemistry of surfaces the method is useful also in biological sciences.

One of the clones of SPM is Atomic Force Microscopy (AFM). AFM is based on mapping of an atomic-force field on a surface of an examined sample. Atomic forces are tested using a small tip attached on the flexible cantilever that moves above the surface. Attractive or repulsive forces are detected upon flexion of the cantilever with tip. This flexion is monitored using a sensitive usually laser-based detector. Both conductive and nonconductive samples can be studied this way.

Forces deforming a cantilever can have a different physical origin. Mostly van der Waals attractive forces acting between two atoms on larger scales are dominant. Repulsive forces between charged particles are important for shorter distances.

According to the type of the contact between the tip of the cantilever and the sample AFM can operate in three modes:

1. Contact mode:

Distance between the tip and examined surface is so small (they are in contact), that the overall force is repulsive and tends to flex the cantilever out of the surface.

2. Noncontact mode:

In this vibrational technique, an average force between the tip and surface is attractive. The tip vibrates in the di- rection perpendicular to the surface, value of its amplitude is in order of tens of $\mathrm{nm}$ and resonance frequency is 200 $\mathrm{kHz}$. Because there is no mechanical contact it is possible to measure also soft and elastic samples. Their possible contamination is prevented.

3. Intermitent contact mode:

It resembles the previous mode. Amplitudes of tip vibrations are greater and so the tip is in contact with the surface for a small portion of vibration period. This approach is more convenient than contact mode. It is used when damage of the examined sample could be caused by friction or drawing. It is more demanding than noncontact mode.

AFM provides a real topographical three-dimensional image of a sample surface with vertical resolution from 0.1 $\mathrm{nm}$ and lateral resolution from $0.1 \mathrm{~nm}$. The obtained resolution depends on a given sample. Good samples can provide even atomic resolution. It gives an image in real time and so can be used also for monitoring of dynamic processes. The greatest advantage of AFM applications in biology is the possibility to image biological samples in vitro and in vivo. A special construction of AFM scanner enables to work directly in liquid environments (9).

Artifacts caused by dehydration of samples are eliminated this way. Imaging using AFM is nondestructive and samples can be visualized several times. Physical or chemical fixations as well as coating of surfaces by sputtering for having a better contrast and conductivity are not necessary.

Dentin of human teeth is a vital tissue that contains a large fraction of water and organic matter. Using the stan- 
dard procedure for preparation of dentin samples in SEM (Scanning Electron Microscopy) a sample is dehydrated in graded acetone series, dried and coated in sputtering device after its fixation. The occurrence of artifacts caused by shrinking (15) cannot be eliminated in practice because dentin is strongly sensitive to dehydration.

We have investigated the possibility to examine dentin surface of human teeth under moist conditions using AFM This method that is not based on aggressive chemicals can help in excluding artifacts.

\section{Method}

Human extracted third molars without decay stored in solution of $0.5 \%$ chloramine for the period of less than one month after their extraction at the temperature of 4 degrees of Celsius (according to the rules ISO TR 11 405) have been used. After removing soft tissue and debris anatomical crown and apical part of a root have been separated using a diamond disc. 3-mm high dentin disc has remained after this procedure. A thin layer of cementum on the surface is removed with a low-speed handpiece under water cooling. Then the outer surface is polished by paper discs Sof-Lex (3M ESPE). Every dentin slice is divided into two halves and placed into distilled water in an ultrasonic purified apparatus for 30 minutes. The samples have then been examined by AFM in Laboratory of Atomic Force Microscopy.

AFM Explorer manufactured by ThermoMicroscopes (USA) has been applied using contact mode with tips from silicon nitride (type 1520-00, ThermoMicroscopes). The imaged surface area has gone from 5 to $100 \mu \mathrm{m}$ and resolution of 300 points per row has been used. Maximum

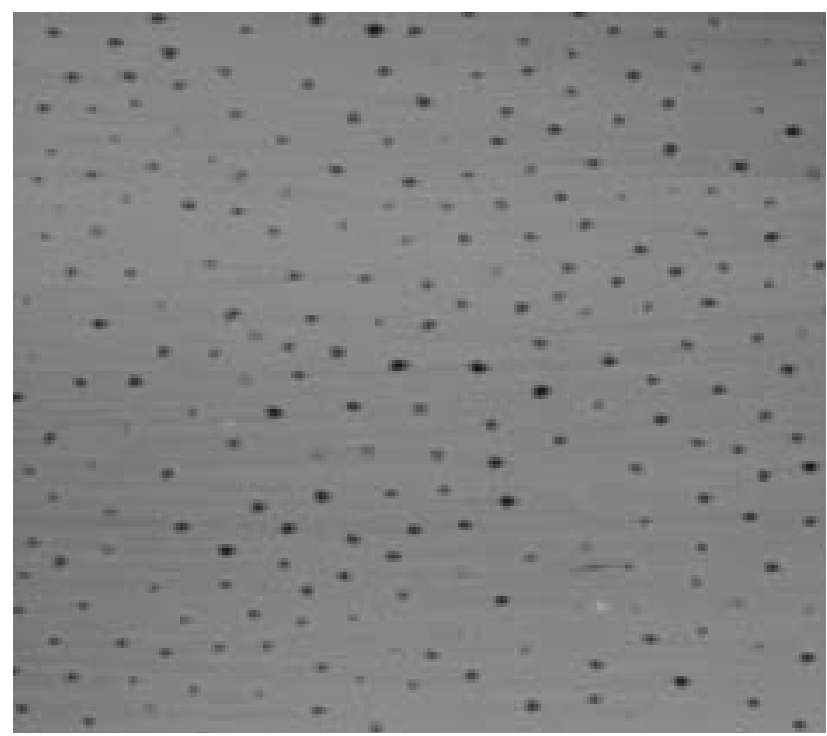

Fig. 1: Picture of a flat dentin surface with opened dentinal tubules without smear layer obtained by AFM (scan area $100 \times 100 \mu \mathrm{m})$. measurable changes of the surface profile have been $10 \mu \mathrm{m}$. Dentin samples have been stored in distilled water and processed under moist conditions.

\section{Results}

Figures 1-4 show dentin surfaces and they also demonstrate problems that have been faced using AFM as a method for imaging. A flat dentin surface with well-shown dentinal tubules without smear layer are depicted in Fig. 1. Dentinal tubules as well as obvious drafts caused when preparing a sample are shown in Fig. 2. Dentinal tubules surrounded by a wall (caused probably by mechanical preparation of the sample) can be seen in Fig. 3. Morphology of a dentinal tubulus is visualized in 3D graph in Fig. 4.

Samples were small and that is why they had to be fixed to a bedding in order to allow for a convenient manipulation.

Natural curvature of a tooth root in two planes and a small roughness occurring on dentin surface after removing cementum complicated imaging and could also lead to a damage of the used tip. This problem has been removed polishing the surface by fine paper discs Sof-Lex under water cooling.

We have had to solve the problem of contamination of sample surfaces. If a surface is not clean enough a lot of strips occur in the image; they are caused by small particles that are moved on a surface by the tip. Removal of smear layer that arises on the surface after its instrumentation and prevents dentinal tubules from imaging is necessary. Removal of smear layer as well as plugs in dentinal tubules can be safely reached polishing the samples and subsequently putting them into an ultrasonic bath for 30 minutes. Removal

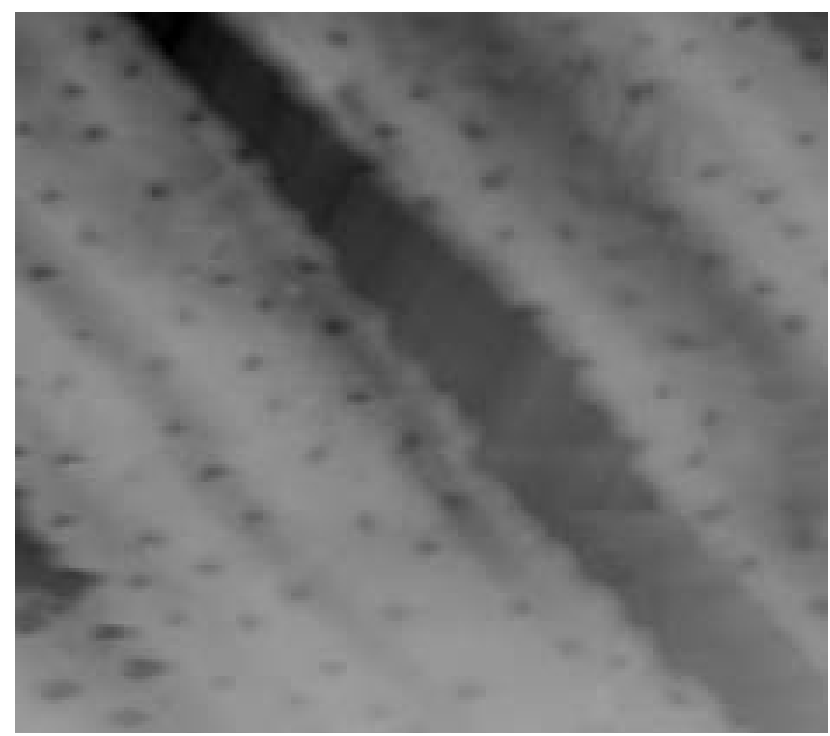

Fig. 2: Visible drafts on a surface of a dentin sample caused by mechanical preparation and depicted by AFM (scan area $100 \times 100 \mu \mathrm{m})$. 


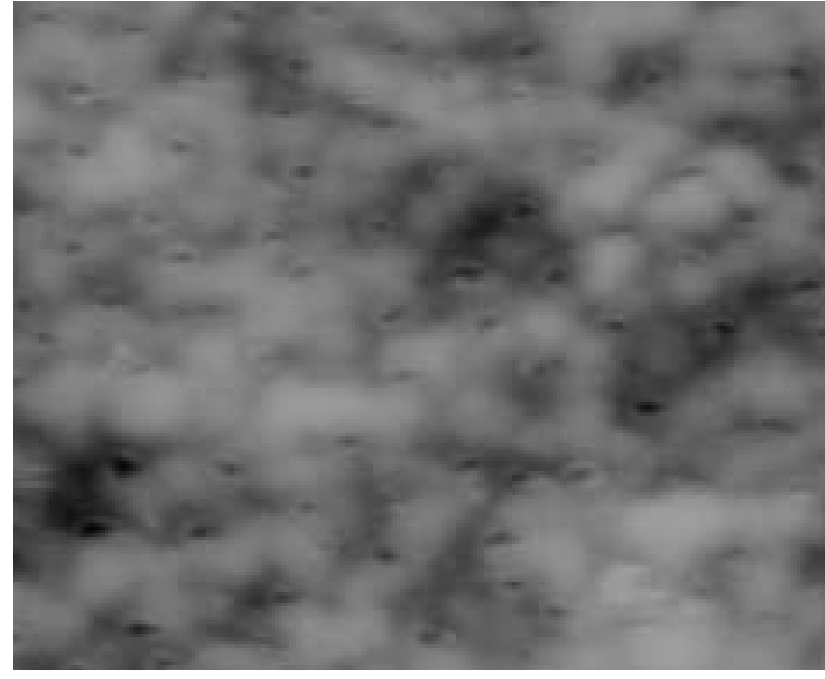

Fig. 3: Dentinal tubules are surrounded by a wall in this image taken by AFM (scan area $25 \times 25 \mu \mathrm{m}$ ).

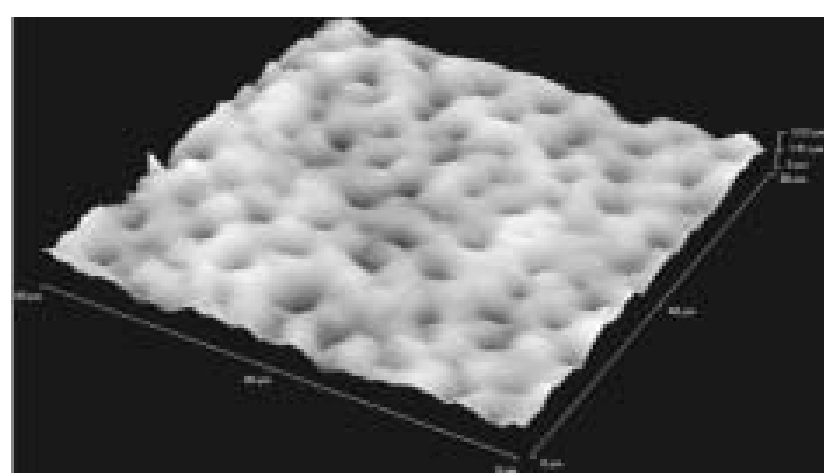

Fig. 4: 3D-image of dentin surface taken by AFM provides full information (scan area $5 \times 5 \mu \mathrm{m}$ ).

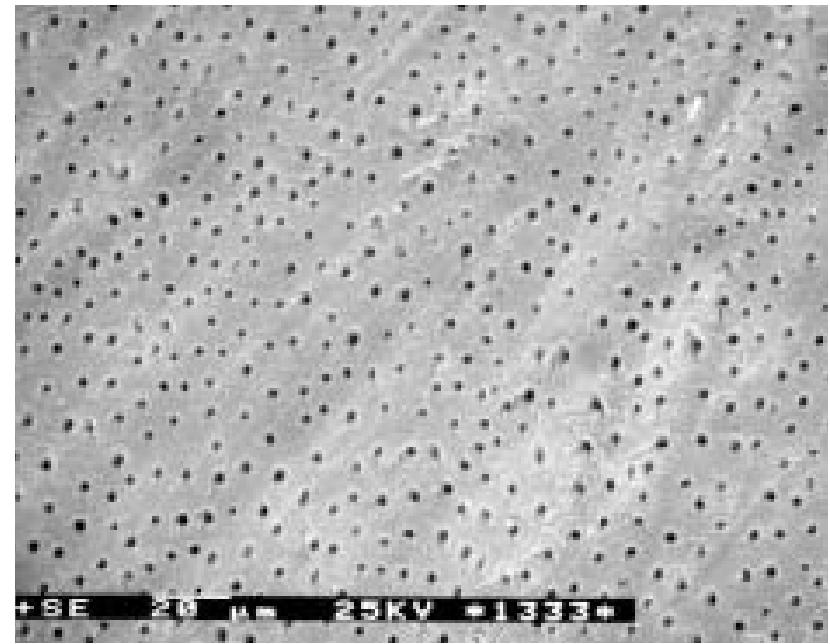

Fig. 5: Dentin surface with open dentinal tubules as seen by SEM; visible drafts remind mechanical preparation; (original magnification x1000, bar represents $20 \mu \mathrm{m}$ ). of smear layer applying $37 \%$ solution of phosphoric acid or combining phosphoric acid with natrium hypochloride lead to demineralization, event. deproteination of dentin.

Disadvantage of AFM approach is the fact that taking an image requires approx. five minutes. We obtain information about the sample surface just bellow the tip that covers a small area. Information about the rest of a sample surface is missing owing to a small scanned area and this complicates statistical analysis of the surface. Samples have to be moved mechanically bellow the tip which also causes troubles in a real experiment. To compare with Scanning Electron Microscopy (SEM), this method enables to visualize a large area of the surface and a subsequent enlargement of resolution is possible. We show pictures of surface - dentin morphology obtained by SEM for comparison. Samples have been prepared using a standard method for Scanning Electron Microscopy (dehydration in a grated acetone series, drying in CPD-030, coatinge by a 5-nm layer of gold and palladium). Observation has been done using microscope SEM Tesla BS 340. Surface of a dentin sample with well shown tubules is shown in Fig. 5; also evidence about mechanical instrumentation is present. Apertures of tubules are without a smear layer. Surface of dentin shown under a higher resolution is depicted in Fig. 6. Apertures of tubules are mostly covered by smear layer, presence of surface distortions caused by instrumentation is visible.

\section{Discussion and Conclusions}

It is evident from literature that AFM is a frequently used method for imaging of dentin. It is mainly useful in studies of a collagen network of dentin and its changes caused by different chemical agents to dentin $(3,15)$. Changes in intertubular and peritubular dentin caused by interaction with phosphoric acid, self-etching primers, conditioners

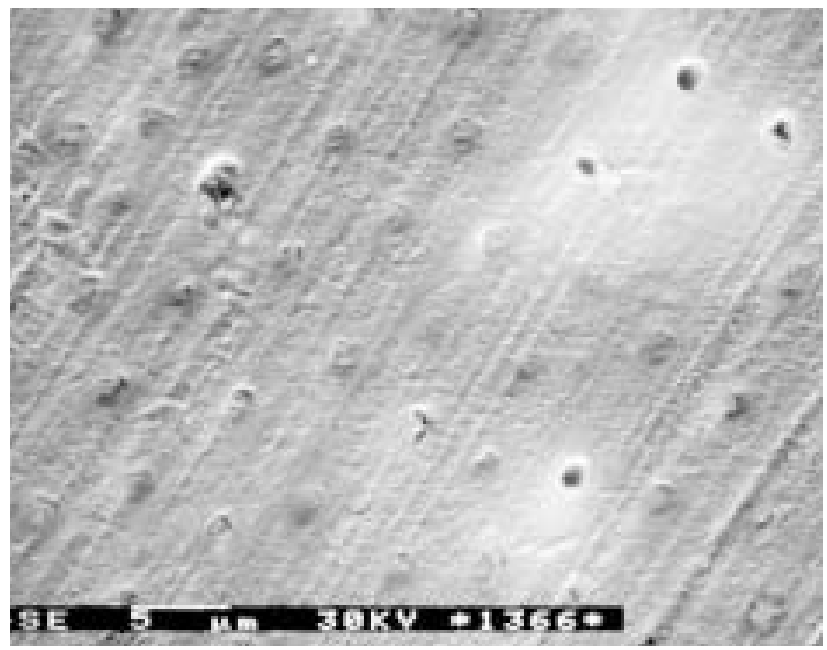

Fig. 6: Dentin surface with concealed apertures of dentinal tubules (original magnification $\mathrm{x} 5000$, bar represents 5 $\mu \mathrm{m})$. 
and other agents used in bonding of restorative materials $(2,11,14,17,19)$ can be investigated. Also interaction of dentin adhesives with tooth hard tissues can be observed $(1,10,12)$.

AFM has been applied in investigations of ultramorphology of superficial and deep dentin and its mechanical properties $(6,15)$. Roughness and elasticity of hydrated peritubular and intertubular dentin has been followed (7). Atomic Force Microscopy enables to observe micromorphology noncarious cervical lesions (13) as well as functional width of the dentino-enamel junction (4). This method also provides information about dentin roughness (16) and enables to register changes in nanomechanical properties of dentin during its storage (5).

We would like to note at this point that AFM has also some limitations. An inevitable use of a cantilever results in many difficulties and restrictions in measurements and sample preparation (20). Furthermore, the high cost of AFM systems prevents their widespread industrial and clinical use, and they are difficult to produce in laboratories that do not specialize in AFM technology (20). When a tip scans across a sample it induces a dynamic interaction force between the tip and the surface (18). The dynamic behavior is complicated and a precise analysis is difficult, but it can influence resolution of the surface image (18).

According to our experience AFM method is not suitable for the estimation of properties of larger areas on a surface. It is time-consuming and requires a flat surface that is accessible to a tip of an AFM microscope. This means a rather strong restriction to flatness of a sample as a whole as well as that concerning flatness of its detailed parts. It is without doubts that AFM brings new possibilities in imaging of dentin surfaces. For this reason, further spreading of this nondestructive method is expected in near future. We can see its main advantage in the fact that this method enables to study wet and chemically non-modified surfaces and so it does not faces troubles connected with artifacts caused by dehydration as the other methods do.

This work is supported by the project by IGA no. NK/7710-2/2003 of the Ministry of Health Care of the Czech Republic (Influence of Nd:YAG laser irradiation of cervical dentin of extracted human teeth).

\section{References}

1. Cassinelli C, Mora M. Atomic force microscopy studies of interaction of a dentin adhesive with tooth hard tissue. J Biomed Mater Res 1994;28:1427-31

2. El Feninat F, Ellis TH, Sacher E et al. A tapping mode AFM study of collapse and denaturation in dentinal collagen. Dent Mater 2001;17:284-8.

3. Habelitz S, Balooch M, Marshall JS et al. In situ atomic force microscopy of partially demineralized human dentin collagen fibrils. J Struct Biol 2002;138: $227-36$

4. Habelitz S, Marshall SJ, Marshall GW Jr et al. The functional width of the dentino-enamel junction determined by AFM based nanoscrating. J Struct Biol 2001;135:249-301.

5. Habelitz S, Marshall GW Jr, Balooch M et al. Nanoindentation and storage of teeth. J Biomech 2002;35:995-8.

6. Kinney JH, Balooch M, Marshall GW et al. A micromechanics model of the elastic properties of human dentine. Arch Oral Biol 1999;44:813-22.

7. Kinney JH, Balooch M, Marshall GW et al. Hardness and Young's modulus of human peritubular and intertubular dentine. Arch Oral Biol 1996;41:9-13.

8. Kubínek R, Vůjtek M, Mašlán M. Mikroskopie skenujicí sondou. Olomouc: Vydavatelství UP Olomouc, 2003:145p.

9. Kubínek R, Vůjtek M, Holubová R et al. Biologické aplikace AFM. Čes čas fyz 2003;53(2):109-12.

10. Kwon TY, Imai Y. Effect of ferric chloride/citric acid/phosphoric acid conditioner on adhesion of 4-META/MMA-TBB resin to the tooth. Dent Mater J 1999; 18:184-93.

11. Marshall GW, Saeki K, Gansky SA et al. AFM study of citric acid-ferric chloride etching characteristics of dentin. Am J Dent 1999;12:271-6.

12. Marshall GW Jr, Marshall SJ, Kinney JH et al. The dentin substrate: structure and properties related to bonding. J Dent 1997;25:441-58.

13. Marshall GW Jr, Chang YJ, Saeki K et al. Citric acid etching of cervical sclerotic dentin lesions: an AFM study. J Biomed Mater Res 2000;49:338-44.

14. Oliveira SS, Marshall JS, Hilton JF et al. Etching kinetics of self-etching primer. Biomaterials 2002;23:4105-12

15. Perdigao J, Thompson J, Toledano $\mathrm{M}$ et al. An ultra-morphological characterization of collagen-depleted etched dentin. Am J Dent 1999;12:250-5.

16. Rosales JI, Marshall GW, Marshall SJ et al. Acid-etching and hydration influence on dentin roughness and wettability. J Dent Res 1999;78:1554-9.

17. Tanumiharja M, Burow MF, Cimmino A et al. The evaluation of four conditioners for glass ionomer cements using field-emission scanning electron microscopy. J Dent 2001;29:131-8.

18. Tser-Son Wu, Win-Jin Chany, Jung-Chang Hsu. Effect of tip length and normal and lateral contact stiffness on the flexural vibration response of atomic force microscope cantilevers. Microelectr Eng 2004;71:15-20.

19. Van Meerbeek B, Yoshida Y, Snauwaert J et al. Hybridization effectiveness of a two-step versus three-step smear layer removing adhesive system examined correlatively by TEM and AFM. J Adhes Dent 1999;1:7-23.

20. Yoshinobu M, Sadao O. Fabrication of micro tactile sensor for the measurement of micro-scale local elasticity. Senzors Actuators A 2004;109:202-7.

MUDr. Zdeňka Zapletalová,

Palacky University Olomouc,

Faculty of Medicine, 1st Clinic of Dentistry, Palackého 12, 77200 Olomouc, Czech Republic. e-mail: zdenazap@tunw.upol.cz 